\title{
Der hohe Staudenrittersporn - Karl Foersters „Blauer Schatz der Gärten“ und „Ritter der Rose“ \\ Hermann Oehring
}

\begin{abstract}
Larkspurs (Delphinium) are attractive ornamental perennials for the garden. They are especially elegant in combination with roses. Hints for culture and use as garden plant are given. The relation between Delphinium and the famous gardener and breeder Karl Foerster from Potsdam-Bornim is outlined.
\end{abstract}

\section{Zusammenfassung}

Rittersporne (Delphinum) sind attraktive Zierstauden für den Garten. Sie gelten als elegante Begleiter von Rosen. Es werden Hinweise zur Kultur und Verwendung des Rittersporns gegeben. Die Beziehung des berühmten Gärtners und Pflanzenzüchters Karl Foerster aus Potsdam-Bornim zum Rittersporn wird erläutert.

\section{Rittersporn als attraktive Begleitstaude} Wenn ab Mitte Juni in zahlreichen Bauerngärten die hochaufragenden Blütentürme der Rittersporne ihr prachtvolles Blau in vielen Farbabstufungen entfalten, wird man sich bewusst, warum Karl Foerster dem Rittersporn den „Königsthron der blauen Farbe im Garten“ zugedacht hat. Der berühmte Altmeister der Stau-

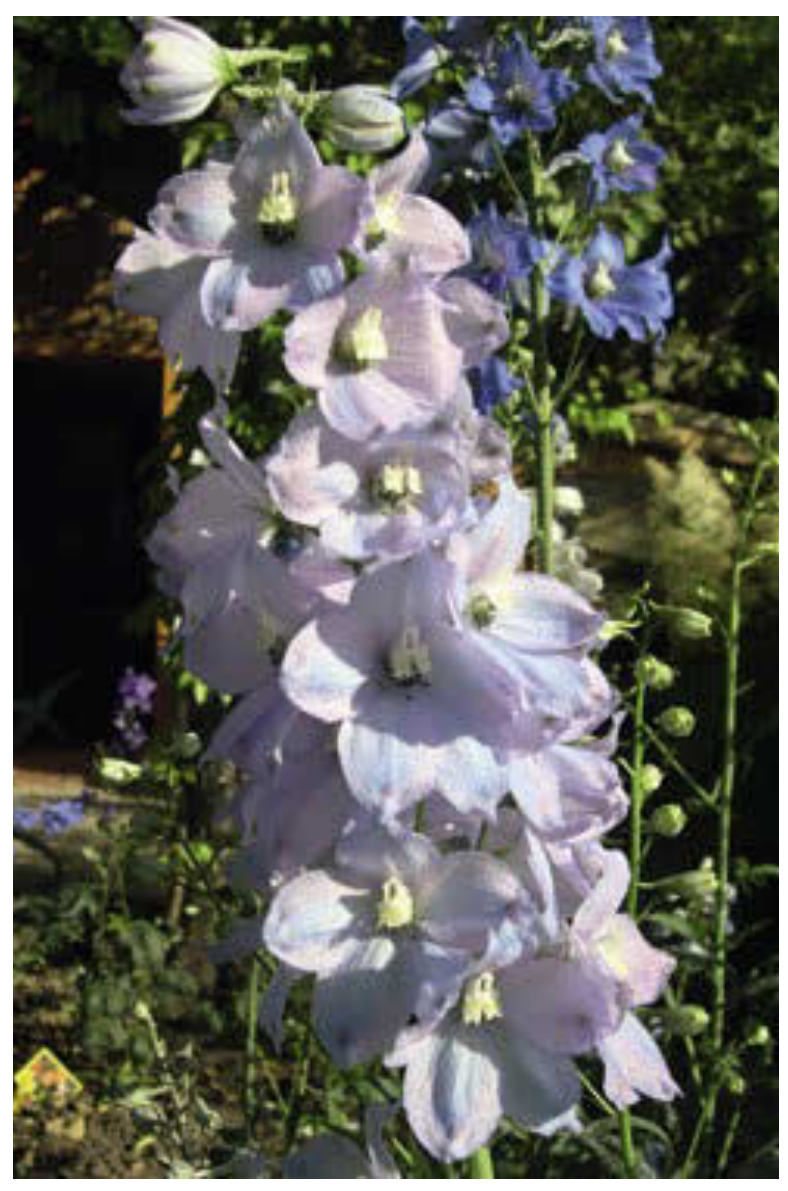

denzüchtung gilt zu Recht als Vater der modernen Rittersporne. Frühzeitig hat Foerster das ungeheuer große Potenzial dieser frühsommerlichen Prachtstaude bei der Gestaltung der Gärten erkannt. Er würdigt den Rittersporn und seine Verwendung im Garten folgendermaßen:

„... Rittersporn ist merkwürdig vielseitig im Garten verwendbar, sofern dort auch seine zeitweise störende Wirkung nach dem Abblühen und das Auf und Ab seiner Blütezeiten vertragen wird. Der naturgegebene Landschaftshintergrund dieser Bergstaude ist die Fichte und Tanne. In Naturgartenpartien, also auch am Rande von Steingärten, kommen nur einfachblühende, nicht allzu gartenhafte Sorten in Betracht, am besten in reinem Hellblau und Azurblau. Gute Nachbarschaft halten andere Bergblumen, so die Mittsommermargerite, sodann Feuerlilien, hohe weiße Glockenblumen, Königskerzen und auch gelber Felberich. Aber auch Rosen im Wildartencharakter und von passender Blütezeit können bei dieser Aufzählung nicht ungenannt bleiben..." (Foerster 1969, S. 106). Auch zur Vergesellschaftung mit Rosen hatte Foerster seine klaren Vorstellungen:

Abb. 1 (links): Delphinium 'Augenweide'.

Abb. 2 (Seite 115 oben): Delphinium 'Abgesang' auf der Freundschaftsinsel in Potsdam.

Abb. 3 (Seite 115 unten): Delphinium-Hybride 'Klingsor'. 
Außerhalb des Bereiches der Wildnisgartenkunst ist natürlich die Rose die eigentliche Partnerin des Rittersporns. Kein Rosarium ohne Rittersporn! Keine Ritterspornpflanzung im Großen ohne ausgewogene Rosennachbarschaft! Da es aber frühblühende und spätblühende Rittersporne wie auch frühe und späte Rosen jeder Art gibt, so ist hier der Erprobung und Dauererfahrung unter allen Umständen nicht nur ein weites, sondern unendliches Feld eröffnet, da ja auch immer neue Rosen und Rittersporne gesteigerter Eigenschaften hinzutreten. Dem Zusammenspiel von Rose und Rittersporn, das sich im nachsommerlichen Flor der beiden noch einmal wiederholt, soll man die Nachbarschaft raumentsprechender Nadelgehölze nicht versagen. Eine Menge von Nadelhölzern, die im Laufe mehrerer Jahrzehnte noch nicht einmal zimmerhoch werden, warten auf Einlaß in Gärten der mannigfachsten Art" (Foerster 1953, S. 31). Geht man nun heutzutage in der recht umfangreichen Gartenliteratur, insbesondere solche, die sich mit der Thematik Gartengestaltung mit Rosen beschäftigt, auf die Suche nach dem Rittersporn, so wird man kaum fündig, allenfalls nimmt der Rittersporn bei der Besprechung und Auswahl von Rosenbegleitstauden eine unbedeutende Fußnote ein. Es wird selten ausführlich auf seine erstrangige Rolle als Rosenbegleiter eingegangen. Dies hat sicher Ursachen, die es zu ergründen gilt. Bevor ebenfalls nachzufragen ist, warum bei vielen Gartenfreunden die Kultur des Rittersporns offensichtlich auf große Probleme stößt, soll die Staude kurz vorgestellt werden.

\section{Die Gattung Delphinium}

Der Gattungsname Delphinium bezieht sich auf die Form der Blütenknospen, die an einen Delphin erinnert. Die Gattung Delphinium gehört zu den Ranunculaceae und umfasst etwa 250 Arten, darunter Ein- und Zweijährige sowie Stauden. Sie sind weltweit in gebirgigen Regionen der Nordhalbkugel verbreitet. Eng verwandt mit dem Rittersporn ist auch der Eisenhut (Aconitum). Rittersporne und Eisenhüte sind in allen Pflanzenteilen stark toxisch. Wichtigste Wirkstoffe sind die für den Menschen
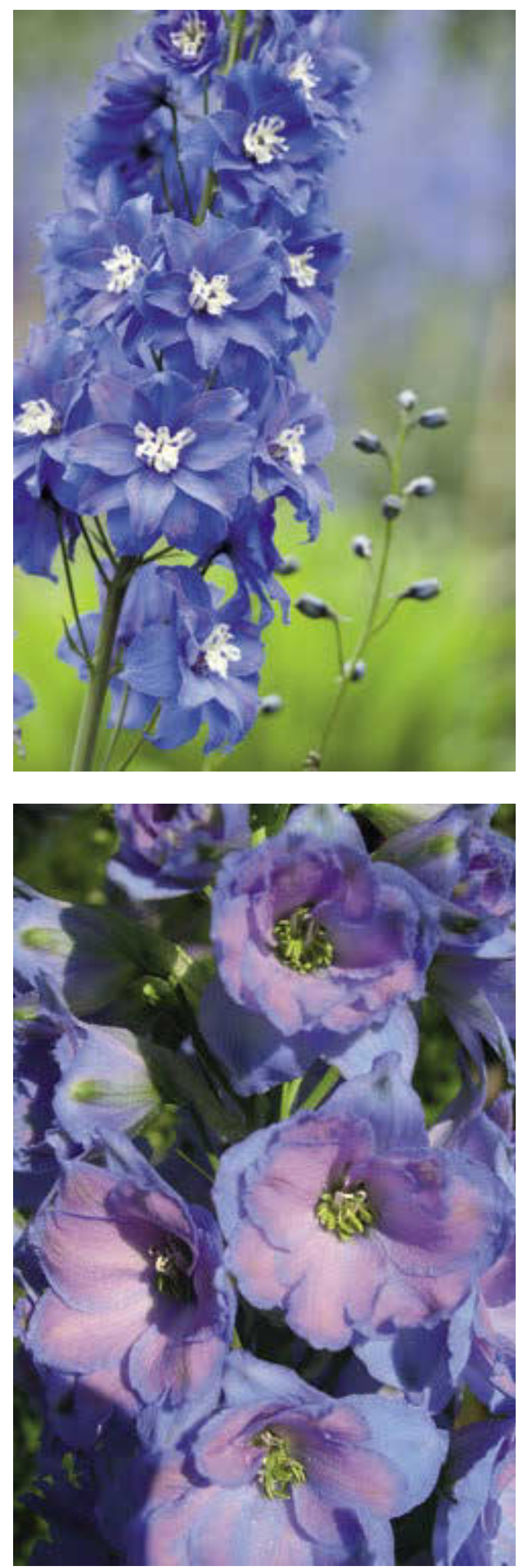


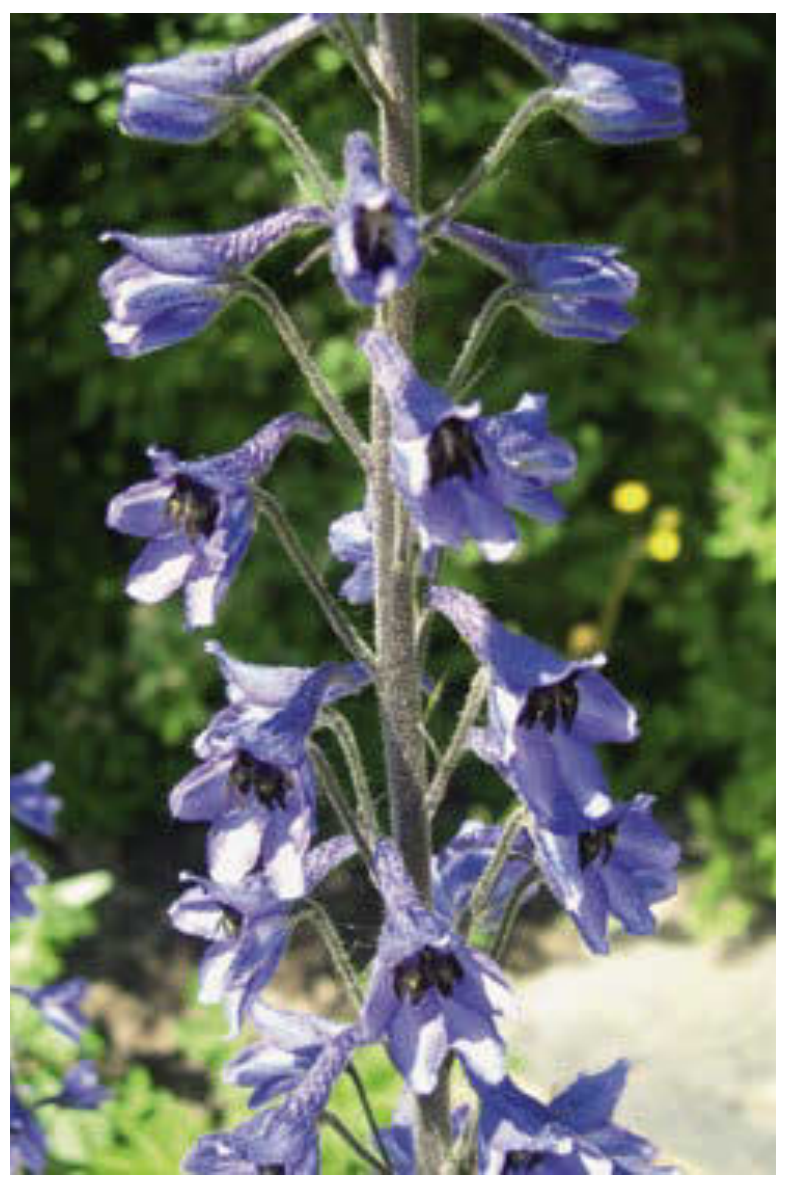

meist extrem giftigen Alkaloide, die oft herzschädigend wirken. Dieser Aspekt sollte bei der Verwendung dieser Pflanzen unbedingt beachtet werden, sodass die Pflanzen beispielsweise nicht in die Hände kleiner Kinder gelangen.

\section{Herkunft und Züchtungsarbeit im 20. Jahrhundert}

Die genaue Herkunft der seit dem 17. Jh. in Kultur genommenen Rittersporne bleibt weitgehend im Dunkeln. Karl Foerster geht davon aus, dass Delphinium. elatum und D. alpinum (aktuell $=D$. elatum) die Stammeltern der heutigen Gartenrittersporne sind. Sie sind in den Ost- und Zentralalpen, in den Pyrenäen und Karpaten, im Riesengebirge sowie in Sibirien und Zentralasien heimisch. Foerster nennt außerdem noch $D$. grandiflorum aus $\mathrm{Si}$ birien und $D$. sinense als für den Garten geeignete Arten. Die aktuelle Nomenklatur fasst beide Arten inzwischen zu Delphinium grandiflorum zusammen. Die typischen Wildstandorte sind lichte Wälder, subalpine Hochstau- denfluren und Bachränder in Höhenlagen bis $2000 \mathrm{~m}$.

Im beginnenden 19. Jahrhundert haben französische und englische Züchter bereits erste Auslesen von Ritterspornen herausgebracht, auf deren Grundlage Karl Foerster zu Beginn des 20. Jahrhunderts seine Züchtungsarbeit aufgebaut hat. Da sich englische Züchtungen in unserem Klima nicht bewährten, hat Foerster besonders die Rittersporn-Auslesen von WILhelm Pfitzer (Stuttgart) in seiner weiteren Zuchtarbeit berücksichtigt. Die Gartensorten sind über lange Zeiträume so vielfältig gekreuzt worden, dass man ihre Eltern nicht mehr erkennen kann. Deswegen werden sie heute als Delphinium-Hybriden (Delphinium x cultorum) zusammengefasst. Dazu gehören die Delphinium elatum-, D. belladonna- und die D. pacificGruppe. Die belladonna-Gruppe geht aus Kreuzungen von D. elatum und D. grandiflorum hervor. Der häufig als Saatgut angebotene hyazinthenblütige Sommerrittersporn gehört zu den einjährigen Arten, die in der Gattung Consolida zusammengefasst worden sind. Diese Rittersporne sollen in der weiteren Betrachtung an dieser Stelle keine Rolle spielen.

\section{Karl Foersters Pionierarbeit in der Ritterspornzucht}

FOeRSTER hat keine systematischen Züchtung in Form einer gezielten Pollenübertragung einer ausgewählten Vatersorte auf eine bestimmte Mutterpflanze durchgeführt, wie es bei vielen anderen Zierpflanzen, auch den Rosen, häufig praktiziert wird. Foerster dagegen hat stattdessen von bereits bestehenden Sorten Saatgut von besonders auffallenden gut gewachsenen Pflanzen gesammelt. Durch Massenaussaaten

Abb. 4 (oben): Wildform von 'Delphinium elatum'.

Abb. 5 (Seite 117 links): Romantische Sommernacht mit Rittersporn.

Abb. 6 (Seite 117 rechts): Garten von Karl Foerster in Potsdam-Bornim, Rittersporn als Begleiter von Rosen; von links nach rechts: Rittersporn in den Sorten 'Finsteraarhorn', 'Tempelgong', 'Polarfuchs' (weiß, halb verdeckt), 'Sopran', 'Morgentau', 'Augenweide', 'Jubelruf'. 


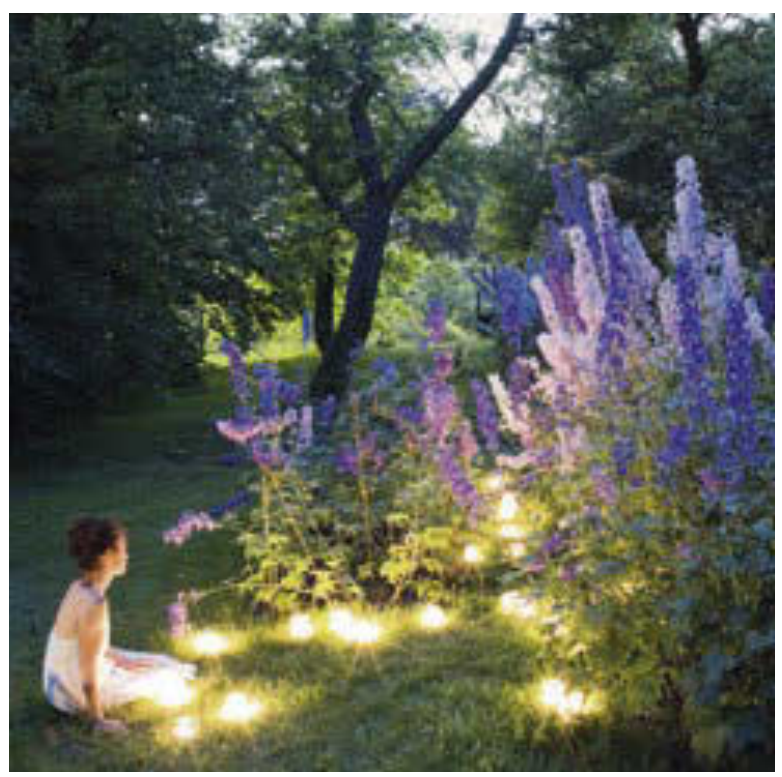

wurden jeweils mehrere Tausend Sämlingspflanzen herangezogen. Seine Genialität als Züchter bestand nun darin, aus dieser Masse erstmals blühender Sämlinge mit unbestechlichem Blick, sein Zuchtziel stets vor Augen, Pflanzen mit herausragenden Eigenschaften auszuwählen. Er handelte stets auch nach dem Voltaire zugeschriebenen Sprichwort, dass das Bessere stets der Feind des Guten sei. So hat FoERSTER immer wieder ältere, von ihm selbst gezüchtete Sorten, die seinen strengen Maßstäben nicht mehr genügten, zugunsten besserer Sorten aus seinem Vermehrungsprogramm herausgenommen.

Karl Foerster legte größten Wert auf Eigenschaften wie Winterhärte, Robustheit, Krankheitstoleranz, Schönheit der Blüte und des gesamten Habitus, klare Blütenfarben, sauberes Verblühen und Langlebigkeit. Die ausgewählten Pflanzen wurden mehrjährigen harten Prüfungen unterzogen (er sprach vom „Enttäuschungsfilter", den die Pflanzen durchlaufen mussten). Und erst, wenn alle Zweifel über die Gartentauglichkeit ausgeräumt waren, wurde die Sorte mit klangvollem Namen getauft und in den Handel gebracht (mündl. Mitt. Marianne Foerster 2009). Häufig hat Karl Foerster aber auch ganz spontan, unmittelbar bei Entdeckung eines besonderen Sämlings, diesem dann einen wohlklingenden Namen gegeben, NäsER (2013) nennt als Beispiel hierfür die Sorte 'Jubelruf'.

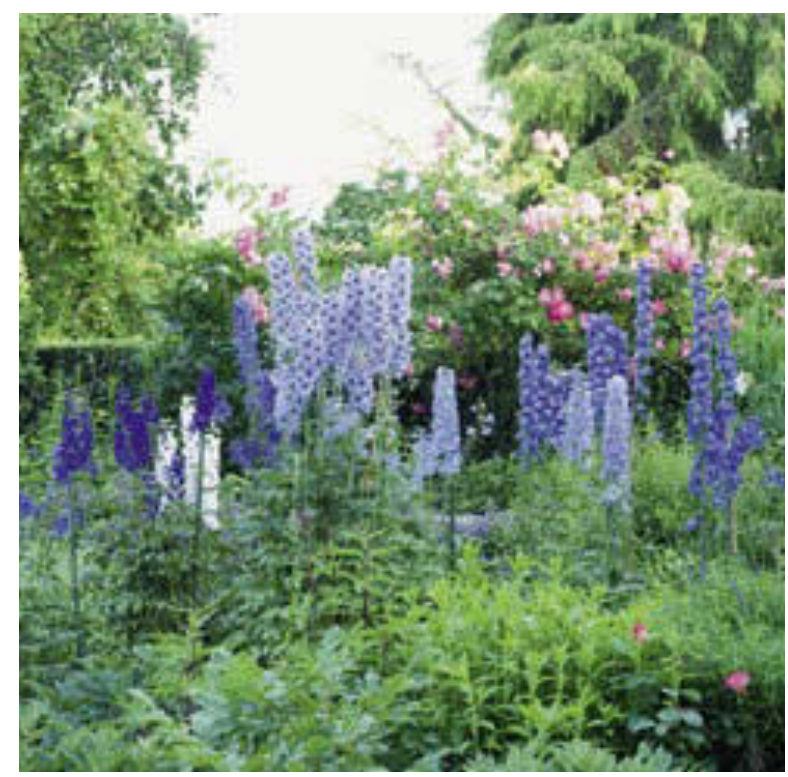

Foersters Delphinium-elatum-Züchtungen haben ihren "Naturadel“ weitestgehend bewahrt, ganz im Gegensatz zu den oft gefüllten fremden Sorten und den üppigen Pacific-Hybriden. Ganz bestimmte züchterisch auszumerzende Fehler bei seinen Ritterspornsämlingen belegte er mit der für ihn typischen bildhaften Sprache. Er gab ihnen humoristische Namen, die sehr gut den entsprechenden Mangel beschreiben:

Strohfeuersorten (Pflanzen, die nach anfänglichem gutem Blühen plötzlich nachlassen), Lückenrispler (Pflanzen, die gehäuft leere Stellen in den Blütenrispen aufweisen), Frostgefährdete (Pflanzen mit verminderter Winterhärte), Geizhälse (Pflanzen, die auch nach Jahren nur sehr wenige Blütenstiele hervorbringen), Wegelagerer (Pflanzen, die ohne zusätzliche Stützung nicht auskommen und umfallen) und Müllerburschen (Pflanzen, die sehr oft und zeitig von Mehltau befallen werden, Foerster 1953).

Letzteres Zuchtziel wurde zu FoERsters Lebzeiten übrigens nie ganz erreicht, erst seinem Schüler und langjährigen Mitarbeiter Wolfgang Kautz in Potsdam-Bornim ist das mit der Selektion der Sorte 'Augenweide' 1981 gelungen. Es handelt sich um eine besonders standfeste, vitale und weitgehend mehltaufreie Pflanze, die sich sehr bewährt hat und auf Gartenschauen mit zahlreichen Preisen bedacht 

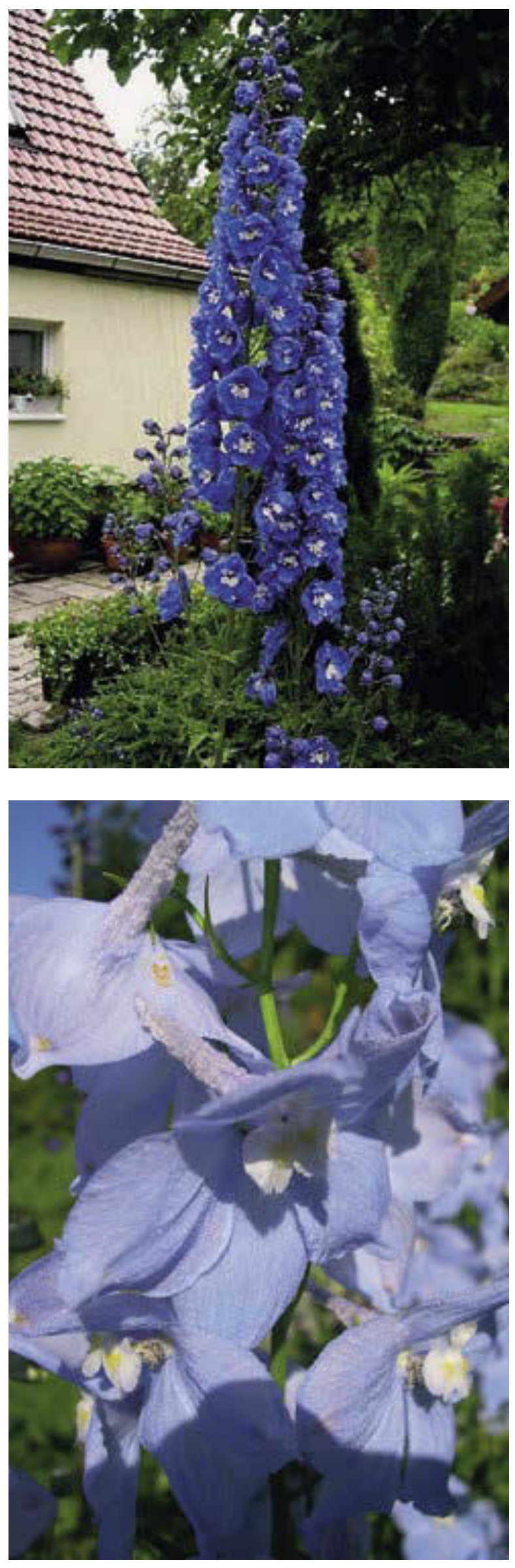

wurde. Von den insgesamt 158 Delphinium-xcultorum-Sorten, die FoERSTER ausgelesen hat, sind noch immer (Stand 2013) 24 Sorten im Standardsortiment, was ein Beleg für die herausragenden Eigenschaften und die Gartentauglichkeit seiner Züchtungen ist.

$\mathrm{Zu}$ den bekanntesten gehören u. a. 'Berghimmel' (1920), 'Gletscherwasser' (1928), 'Finsteraarhorn' (1936), 'Tempelgong' (1936), 'Perlmutterbaum' (1938), 'Tropennacht' (1955), 'Jubelruf' (1956), 'Zauberflöte' (1956), 'Sternennacht' (1961), 'Kleine Nachtmusik' (1964), 'Sopran' (1966), '.Abgesang' (1967) und 'Ariel' (1967). Die Jahresangaben variieren allerdings bei verschiedenen Autoren, NäSER (2013) nimmt als verlässliche Grundlage die alten Foerster-Kataloge, in denen übrigens nur 72 Sorten aufgelistet sind, die jemals in den Handel gekommen sind.

\section{Weitere Züchtungen und ihre Garteneignung}

In Großbritannien, im Mutterland der Gartenkultur, erfreut sich der Rittersporn noch immer sehr großer Beliebtheit, es existiert sogar eine eigenständige Rittersporngesellschaft, die British Delphinium Society. Zahlreiche Sorten werden noch immer selektiert, viele von auserlesener Schönheit und Eleganz, doch sind sie fast alle für unser kontinentales Klima ungeeignet, d. h. nicht ausreichend winterhart, weshalb an dieser Stelle nicht weiter darauf eingegangen werden soll.

\subsection{Rittersporne der Belladonna-Gruppe}

Sie sind von besonderer Wuchsform, wesentlich kleiner und zeigen einen sehr lockeren Blütenstandsaufbau. Sie sind besonders geeignet in gemischten Rabatten (Mixed Borders).

KöHLein \& Menzel (1994) nennen zwei Theorien zur Entstehung dieser Gruppe, wobei beide hypothetisch und nicht nachweisbar sind.

Abb. 7 (links): Pacific-Hybride 'Blue Bird'.

Abb. 8 (rechts): Delphinium-Hybride 'Ballkleid'.

Abb. 9 (Seite 119): Garten der Familie ZAHN in Sulzbach-Rosenberg mit Ritterspornen im Morgenlicht. 


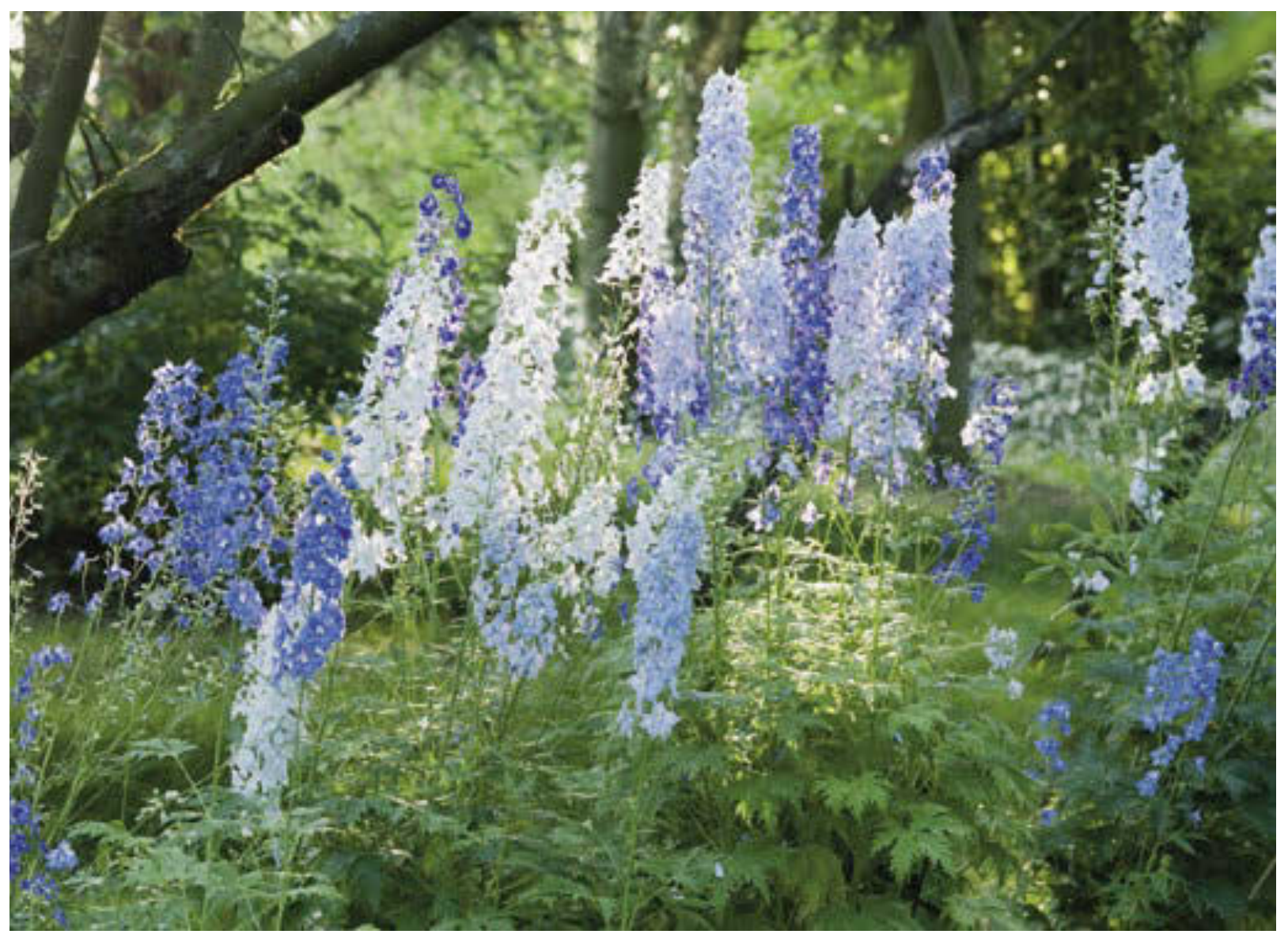

1. Kreuzung zwischen D. elatum und $D$. grandiflorum.

2. D. cheilantum aus Ostsibirien soll zu den Stammeltern gehören.

\subsection{Rittersporne der Pacific-Gruppe}

Wenn Karl Foersters Meinung, dass die Ritterpsorn-Sorte das Schicksal des Gartens beeinflusse, stimmen sollte, so trifft das ganz besonders für die neuerdings sehr populäre Gruppe der Pacific-Hybriden zu. Diese Sorten, ursprünglich in Amerika gezüchtet, meist gefüllt und von kräftiger Farbe, sind ein gutes $\mathrm{Ge}-$ schäft für den Erwerbsgärtner, da leicht aus Samen vermehrbar und bereits im ersten Jahr mit einer Vielzahl von Blütenrispen bestückt. Jedoch sind sie leider nur sehr kurzlebig und eigentlich nur als Einjahresblumen zu verwenden. Kurz gesagt: eine echte Enttäuschung für den wahren Ritterspornliebhaber. Hier liegt vielleicht der Schlüssel, warum so viele Gartenbesitzer nach anfänglicher Begeisterung für diese Staude wieder davon abkommen. Da lei- der aus Unkenntnis alle Rittersporne gewissermaßen in einen Topf geworfen werden, sind Fehlschläge vorprogrammiert und Aufklärung ist hier dringend geboten.

In Gartenmärkten werden inzwischen fast nur noch Pacific-Sorten angeboten. Die meisten Staudengärtnereien verzichten auf die aufwendige und kostenintensive vegetative Vermehrung der Delphinium-elatum-Sorten. Darüber hinaus belegen eigene Erfahrungen, dass aus Gärtnereien bezogene Jungpflanzen selten noch die Sortenreinheit garantieren.

Nach wie vor gilt: Erste Wahl sind noch immer die Züchtungen Foersters (aber natürlich auch diejenigen von Klose, Weinreich, Marx, Kayser \& Seibert und anderen). Verschwiegen werden sollte aber auch nicht, dass die alten bewährten Sorten, die jahrzehntelang durch Teilung vegetativ vermehrt wurden, an Vitalität verlieren und bereits Abbauerscheinungen zeigen.

Darauf hat unlängst auch NÄSER (2013) aus Potsdam-Bornim aufmerksam gemacht. Er 


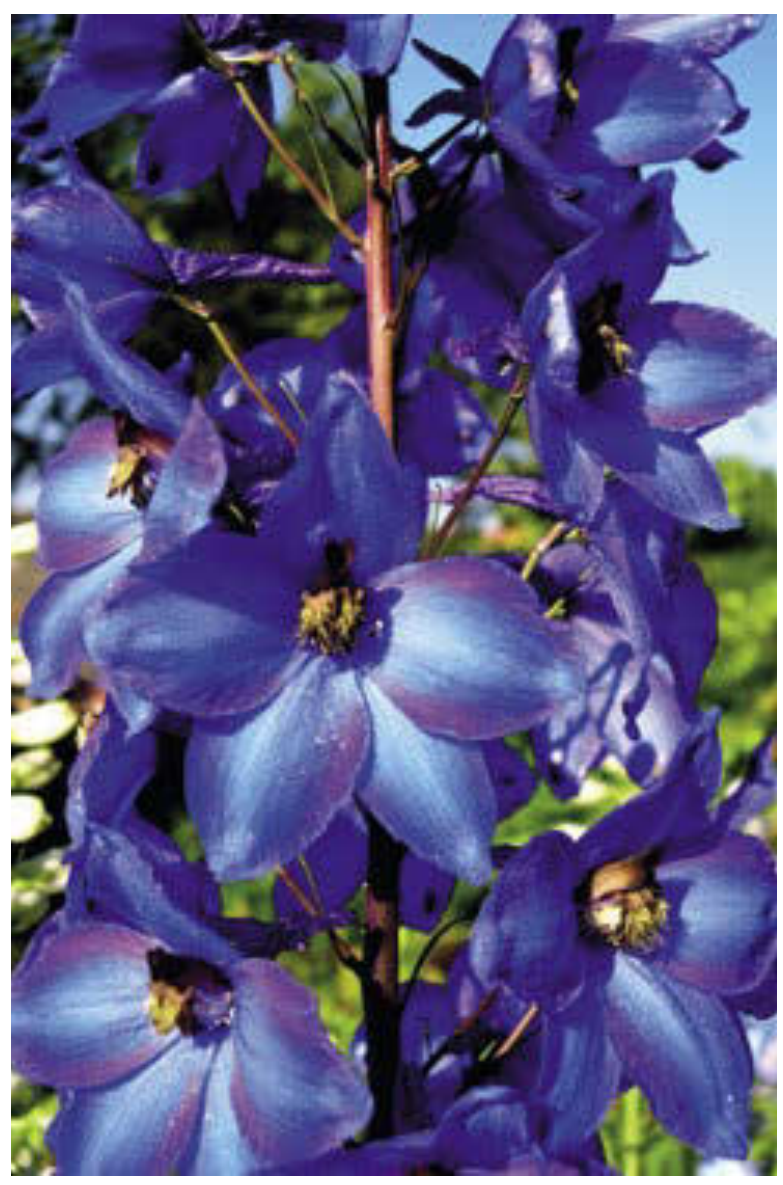

hat in jahrzehntelanger Tätigkeit an der Seite Karl Foersters in der Gärtnerei gearbeitet und viele Neuzüchtungen begleitet. Den zu beobachtenden Rückgang der Lebenskraft (und aus meiner unmittelbaren Erfahrung auch Änderung der Blütenfarbe und des Habitus) bislang bewährter Sorten hat er besonders deutlich gemacht.

\subsection{Hinweise und Ratschläge zum erfolg- reichen Gärtnern mit Rittersporn}

Rittersporn stellt zunächst keine allzu großen Ansprüche an den Boden. Der Boden sollte tiefgründig, lehmig, mit hohem Humusanteil sein und ein gutes Wasserspeichervermögen aufweisen. Nur reinen Sandboden mag er nicht. Als Starkzehrer, der zweimal jährlich ein umfangreiches Blattgerüst aufzubauen hat, benötigt er ein nährstoffreiches Substrat. Dankbar ist Rittersporn deswegen für zusätzliche Kompostgaben, auch gut verrotteter Pferdeund Rinderdung ist geeignet. Ansonsten eignen sich auch Hornspäne und granulierter
Rinderdung aus der Tüte. Nährstoffmangel gibt die Pflanze durch verminderte Wuchsleistung und geringen Blütenansatz zu erkennen. Das Laub verfärbt sich hellgrün und die unteren Bereiche der Pflanze verlieren die Blätter, was auch sehr schnell bei Wassermangel passiert. Überhaupt reagiert der Rittersporn sehr empfindlich auf Wassermangel, da er nur ein flaches Wurzelsystem ausbildet. In Trockenperioden muss also kräftig und durchdringend gewässert werden.

Der Standort sollte sonnig sein, jedoch wird auch Halbschatten toleriert. Da die zarten Triebe eine große Anziehungskraft auf Nacktschnecken ausüben, ist in gefährdeten Regionen unbedingt eine Abwehr durch umweltverträgliches Schneckenkorn zu organisieren. Ganz besonders wichtig ist diese Maßnahme im Frühjahr, unmittelbar nach der Schneeschmelze, da Schnecken bereits $\mathrm{ab}+3{ }^{\circ} \mathrm{C}$ aktiv werden und gezielt die unterirdischen Triebe aufsuchen. Verpasste Schneckenabwehr führt dazu, dass abgenagte Triebspitzen ersetzt werden müssen und die Pflanze dadurch stark geschwächt wird, was zum Totalverlust führen kann.

\subsection{Pflanzung, Pflege und Teilung}

Auch hier ist es angebracht, die gärtnerischen Erfahrungen des Altmeisters zu nutzen. Er schreibt: „Alles in allem ist der Rittersporn in seiner Anpassung an den Boden eine gutmütige, mit rührenden fakirhaften Dauerkräften begabte Kreatur. Man kann ihn in gleicher voller Kraft in Dresdens wie in Hamburgs Gärten, in Meeresstrandgärten vor der Brandung und oben in tausend Meter hohen Berggärten unter beschneiten Felsterrassen ... und Moorbodengärten der Heide blühen sehen." (Foerster 1929, S. 34)

Wenn auch Foerster meinte, eine Teilung frühestens nach 5-6 Jahren vornehmen zu müssen, können eigene Erfahrungen dieses nicht bestätigen. Um Wuchskraft und Vitali-

Abb. 10 (Seite 120): Delphinium-Hybride 'Finsteraarhorn'.

Abb. 11 (Seite 120): Delphinium-Hybride 'Tempelgong'. 
tät zu erhalten, wird eine Teilung nach spätestens 3 Jahren empfohlen, immer unter der Voraussetzung, dass jährlich eine gute Nachdüngung, z. B. mit verrottetem Rinderoder Pferdedung, erfolgt. Dabei hat sich gezeigt, dass der beste Zeitpunkt das zeitige Frühjahr (oder noch besser: Ende Juni nach erfolgtem Rückschnitt) zum optimalen Anwachserfolg führt. Herbstverpflanzung sollte, wie sonst bei Stauden üblich, möglichst vermieden werden, da zu einem späten Zeitpunkt die Wurzelneubildung nur sehr zögerlich erfolgt.

Beim generellen Rückschnitt nach der Hauptblüte, ohne das eine Teilung ansteht, sollten $20-30 \mathrm{~cm}$ Stengelreste verbleiben, da sonst die Kapillarkräfte aus dem Wurzelbereich zu hohen Wasserverlusten führen. Danach ist eine Volldüngergabe sinnvoll, um den Neuaustrieb und die Nachblüte zu fördern. Wer es selbst mit Züchtung und Auslese versuchen will, kann Samenstände von besonders schönen Exemplaren ausreifen lassen. Entscheidend ist dann eine unverzügliche Aussaat, da die Keimfähigkeit des Ritterspornsamens zeitlich sehr begrenzt ist.

\subsection{Vegetative Vermehrung}

Hybridsorten können nicht durch Samen vermehrt werden, sie müssen durch Teilung sortenecht erhalten werden. Ein aufwendiges Verfahren, was aber für den Hobbygärtner reizvoll sein kann. Dabei geht der Autor so vor, dass er den Wurzelstock mit der Grabegabel aus dem Boden nimmt, anhaftende Erde abschüttelt und schließlich in einem Wassergefäß ausspült. Dann werden die Wurzeln um die Hälfte zurückgeschnitten. Geteilt wird mit einem scharfen Messer so, dass an jedem Teilstück mindestens 2 sichtbare Augen bzw. Triebstücke und ausreichend Wurzelmasse verbleiben. Entweder werden die sauber etikettierten Teilstücke wieder direkt in gut humusversorgte Gartenerde gepflanzt. Sie können aber auch in Kultursubstrat unter weitgehendem Verzicht auf Torfzusätze getopft werden. Es bereitet dann viel Freude, andere Gartenfreunde mit Jungpflanzen zu beschenken.

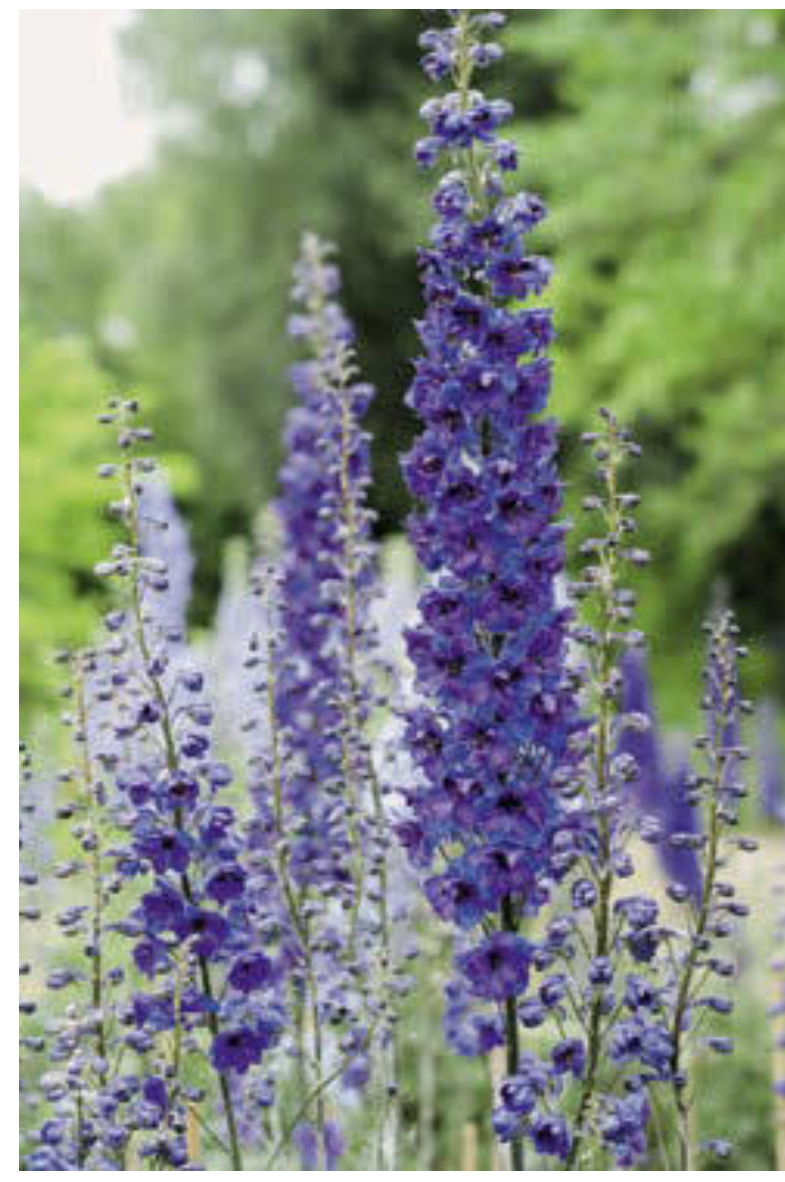

\subsection{Rosen und Rittersporn}

Über die gestalterische Bedeutung beider Partner wurde eingangs schon gesprochen. Da sowohl Rosen als auch Rittersporne zu den Starkzehrern gehören, ist auf genügenden Pflanzabstand, also etwas "Ellenbogenfreiheit" zu achten. Die hoch aufragenden Blütenkerzen der Rittersporne, die die vertikale Dimension besonders betonen, gehören immer in den Hintergrund einer Stauden- oder Rosenpflanzung. Vergesellschaftung mit zwergig wachsenden Nadelgehölzen hatte ja bereits Karl Foerster empfohlen.

Wenn Foerster auch beim Rittersporn von einer besonders langlebigen Pflanze sprach (er kannte ja auch die Spanische Nacktschnecke noch nicht!), so ist eine regelmäßige Verjüngung der Pflanzen ein gutes Rezept für dauerhaften gärtnerischen Erfolg. Allerdings, für den Einsatz im öffentlichen Grün kann Rittersporn aufgrund seines doch recht hohen Pflegeaufwandes nur bedingt für Parkanlagen empfohlen werden. Für gemischte Staudenrabatten je- 
doch und in Kombination mit Klein- und Großstrauchrosen ist der Rittersporn unverzichtbar. Er verdient es deshalb, mehr als bisher, in den Mittelpunkt gärtnerischer Planung und Gestaltung gerückt zu werden.

Ein Foerster-Zitat soll diesen Beitrag abrunden. Unter der Überschrift „Der Rittersporn blüht" schreibt er:

„... die blaue Blumenfarbe ist und bleibt die größte Verzauberin des Gartens und die größte Versöhnerin anderen Blumenfarbenstreits. Sie scheint uns im Garten und gleichermaßen in großen Naturwildnissen ... ein vom Himmel gefallenes Juwell die Herrlichkeit eines Sommermorgens im Garten kann ohne Rittersporn nicht ausgeschöpft werden."(Foerster 1929, S. 34).

Und abschließend Foersters Bekenntnis seiner besonderen Hinwendung zum Rittersporn mit dem berühmt gewordenen Zitat:

„... wer seine ganze Lebensarbeit der blauen Blume, ja nur der schönsten unter ihnen, dem Rittersporn, verschreiben wollte, sähe sich am Ende eines neunzigjährigen Lebens noch vor blauer Unendlichkeit der Aufgaben." (Foerster 1969, S. 104)

Es ist nun wieder an der Zeit, einer der eindrucksvollsten blauen Frühsommerstauden, dem hohen Stauden-Rittersporn (D. elatum und seinen Zuchtsorten), den ihr gebührenden Platz bei der Gartengestaltung einzuräumen. Dieser Beitrag soll dabei helfen.

\section{Dank}

In Memoriam Marianne Foerster gewidmet.

Für die kritische Durchsicht des Manuskripts danke ich den Herren Dr. Konrad NäSER, Potsdam-Bornim, ehemaliger Züchtungsleiter in der Staudengärtnerei Karl Foerster, und Karl Wienke, Suhl, freier Landschaftsund Gartenarchitekt.

\section{Verwendete und weiterführende Literatur}

Foerster, K.1929: Der neue Rittersporn. Geschichte einer Leidenschaft. Reprint 1990. - Sulzberg.

Foerster, K.1953: Blauer Schatz der Gärten. Berlin, Radebeul.

Foerster, K. 1969: Es wird durchgeblüht. 2. Aufl. - Berlin.

Kreuter, M. L. 2006: Karl Foerster, Züchtungen und Gedanken für die Zukunft. - Potsdam. Näser,.K. 2013: Die Suche nach Atlantis GartenFlora 6/2013: 14- 17.

Mehliss, C. 2012: Karl Foerster, seine Blumen, seine Gärten. - Stuttgart.

Reif, J. \& Härtel, W. 2010: Foersters-StaudenKompendium. - Potsdam.

Barthlotт, W. (Hrsg. 2006): Die große Pflanzenenzyklopädie. - London.

Köhlein, F. \& Menzel, P. 1994: Das große Buch der Stauden und Sommerblumen. - Stuttgart.

SEyffert, W. 1981: Stauden für dekorative Gestaltung und Schnittblumengewinnung. - Berlin.

Hansen, R.\& Stahl, F. 1990: Die Stauden und ihre Lebensbereiche. - Stuttgart. GöRITZ, H. 1988:

Blütenstauden, Gräser, Farne. - Berlin. 